\title{
Tobacco and Alcohol Use in Cuban Women
}

\author{
Patricia Varona MD MS, Martha Chang, René G. García MD, Mariano Bonet MD
}

\begin{abstract}
INTRODUCTION Tobacco and alcohol are currently the most widely consumed legal psychoactive substances in the world. They represent a heavy burden for health and society in almost all populations. Increasing consumption of both substances is a trend observed in women.
\end{abstract}

OBJECTIVE Describe the profile of women aged $\geq 15$ years residing in urban areas of Cuba with respect to tobacco and alcohol consumption.

METHOD Basic information on tobacco and alcohol consumption by Cubans aged $\geq 15$ years in urban areas was obtained from the Second National Survey on Risk Factors and Chronic Diseases (2001), a national descriptive cross-sectional study, the objective of which was to determine the frequency and epidemiological characteristics of the urban population's main chronic disease risk factors. Sampling design was complex stratified multi-stage cluster. Of a sample of 23,743 individuals, 22,851 were surveyed, representative of 6.8 million Cubans. A questionnaire and structured interview were used. Variables were tobacco and alcohol use, as well as sociodemographic factors: sex, age, educational level, skin color, marital status, type of full-time employment and perceived economic situation. Prevalence, with 95\% confidence intervals, and male:female prevalence ratios were estimated.

\section{INTRODUCTION}

The most widely consumed legal psychoactive substances in the world, tobacco and alcohol, confer a heavy health burden on almost all populations. Approximately one third of the world's population reportedly smokes; and in developed countries, $80 \%$ of men and $60 \%$ of women have consumed alcohol at some point in their lives, with lower, but still substantial, consumption rates in developing countries.[1-4]

The negative health effects of smoking and alcohol are well known, as are their repercussions on the individual, family and society. Yet, more and more young people are initiating tobacco and alcohol use.[5,6]

The use and abuse of these substances contribute significantly to morbidity, premature mortality, disability and lower quality of life; and are associated with more than 60 conditions affecting health.[7,8] Although risk of disease or death from use of either substance vary according to factors such as amount used chronically, almost all organs of the human body can be affected.[1,7]

There is consensus that a gender differential exists, with women more vulnerable to harm from tobacco and alcohol use.[9-11] Women are more susceptible to tobacco's carcinogenic effects on the lungs and metabolize nicotine differently. With respect to alcohol, women are more susceptible to its damaging effects because the enzymatic processes for oxidizing alcohol in the female liver are slower than in the male's. While men metabolize up to $30 \%$ of alcohol ingested, women metabolize only $10 \%$, and thus have higher blood alcohol levels for the same amount of alcohol consumed.[12,13]

As a result, women with higher intakes of these substances are more likely to have health problems such as complications of
RESULTS Women who smoked were predominantly aged 40-59 years; had completed less than university education; of black skin color; divorced; laborers, service workers or managers, and with a perceived economic situation as very poor. Women who consumed alcohol were predominantly aged $15-59$ years, had at least middle school education, of mestizo or black skin color, of marital status other than widowed; there was no typical profile for occupation or perceived economic situation. Women who were both smokers and alcohol consumers were predominantly 20-59 years, of black or mestizo skin color, of marital status other than widowed; with no typical profile for educational level, occupation or perceived economic situation.

CONCLUSIONS The first nationwide socioeconomic profile of Cuban women using tobacco, alcohol or both constitutes a baseline for comparison to results of a new national study now under way, permitting evaluation of trends over time and effectiveness of prevention and control efforts.

KEYWORDS Risk behavior, risk factors, behavioral risk factor surveillance system, smoking, tobacco smoking, alcohol, alcohol consumption, psychoactive agents, prevalence, women's health, Cuba

pregnancy and increased likelihood of bearing children with disorders such as sudden infant death syndrome and fetal alcohol syndrome. These women are also more likely to suffer from high blood pressure, menstrual cycle alterations and sleep disorders, as well as mental health problems-including depression, stress, anxiety or guilt-that can trigger self-destructive behavior.[14-16]

Problems of tobacco and alcohol use are now being seen in populations that until recently had not been so affected. In recent decades, the social role of women has changed in many societies, and with this has come changes in their patterns of tobacco and alcohol use. Cigarette manufacturers have also deliberately targeted women, another contributing factor to rising rates of smoking among women in many developing countries.[15,16]

Reportedly, at least $10-12 \%$ of women smoke worldwide, although stigmatization of women smoking may cause underreporting in some regions. Tobacco use by women varies geographically: male:female differences are greater in the Pacific region and smaller in Europe and the Americas, where approximately 1 in 4 women smoke. Alcohol consumption in women in this last region is approximately $40 \%$ above the world average. $[5,15]$

Cuba's Second National Survey on Risk Factors and Chronic Diseases (2001) (Spanish acronym, ENFRENT II), a nationwide study of the urban population aged $\geq 15$ years, found smoking prevalence of $23 \%$ in women and $41 \%$ in men.[17]

In $2007,12 \%$ and $3.7 \%$ of all deaths globally were attributable to smoking and alcohol, respectively. Alcohol was responsible for $4 \%$ of the global burden of disability-adjusted years of life lost. In the same year, mortality attributable to tobacco use in Cubabased on data for three disease groups: cardiovascular diseases, cancer, and chronic bronchitis-was estimated at 18\% (15,083 
deaths). Women accounted for one third of these deaths (5027). [18-21]

Several socioeconomic factors in Cuba-including long traditions of tobacco farming and rum production and permissive cultural attitudes-are associated with social acceptance of smoking and alcohol use. Public health strategies through 2015 in Cuba highlight the need for effective prevention and control of use, abuse and addiction related to both these substances.[22,23]

Implementation of sound and sustainable multisectoral interventions regarding alcohol and smoking requires better epidemiological data and analysis. Although local studies provide some information on specific population groups, no national studies have been published specifically addressing these health-related problems in Cuban women, which are necessary to support effective strategies to lower substance-use prevalence.

This article's objective is to describe for the first time the socioeconomic profile of women aged $\geq 15$ years who use tobacco and alcohol, resulting in a baseline to analyze results of the new national study under way, to evaluate trends over time, and the effectiveness of preventive and control strategies.

\section{METHODS}

Data on tobacco and alcohol consumption by Cubans aged $\geq 15$ years resident in urban areas of Cuba (where $75 \%$ of the $\mathrm{Cu}$ ban population lives)[24] were obtained from the Second National Survey on Risk Factors and Chronic Diseases (2001), a descriptive cross-sectional study, to determine the frequency and epidemiological characteristics of the main chronic disease risk factors. [17] While the population of interest was women, data on both sexes were analyzed for comparison purposes.

The survey used a complex stratified multi-stage cluster design prepared by the National Statistics Bureau. The 14 (at the time) provinces were the strata; clusters consisted of sampled geographic areas, blocks and sections.

In each systematically-sampled geographic area, a block was selected in two sections of which all households were visited to interview all permanent residents aged $\geq 15$ years. Sample size was calculated for each stratum to permit stratum-specific estimates; the national sample was formed by aggregating provincial strata. A weighting factor was used consisting of the inverse of individual selection probability, taking into account age and sex, to compensate for possible bias in population estimates. Sampling was equiprobable at the provincial level, but not nationally. Included were 9260 households $(23,743$ individuals) and 22,851 individuals surveyed, $52 \%(11,882)$ of whom were women. Together, they represented 6.8 million Cubans in this age group.[25]

After obtaining informed consent, professional surveyors conducted structured interviews in selected households to gather demographic information on individuals and families, economic conditions, risk perception regarding specific behaviors, selected chronic non-communicable diseases and their risk factors, medication use, violent behavior, disability and preventive practices. This paper reports on the following: sex, age in years (15-19, 2039, 40-59, $\geq 60$ ); educational level completed (primary, middle, high school, university); skin color (white, black, mestizo); marital status (unmarried, married or cohabiting, divorced or separated, widowed); occupation (manager, administrator, upper- or middlelevel technician, laborer, service worker); perceived economic situation (excellent, good, fair, poor, very poor); and tobacco and alcohol use.

Investigators defined tobacco and alcohol use as any use in the 30 days prior to the survey.

Prevalence of tobacco and alcohol use, separately and combined, was estimated for both sexes, although conclusions refer only to women, consistent with the study's objective.

Absolute numbers and percentages were calculated, with $95 \%$ confidence intervals $(\mathrm{Cl})$ around point estimates; differences were considered significant if confidence intervals did not overlap. Percentages were rounded to one decimal place. Male:female prevalence ratios (PR) were calculated. Data were processed using Statistical Analysis Software (SAS) version 8.02, and presented in tables and figures.

\section{RESULTS}

Survey response was high at $96 \%$. Estimated prevalence of smoking in women was $22.3 \%(95 \% \mathrm{Cl} 20.3-24.2)$, for alcohol use $10.4 \%(95 \% \mathrm{Cl} 9.6-11.2)$ and for both $4.0 \%$ (95\% Cl $3.4-$ 4.5). Male:female PR for smoking was 1.8, for drinking 4.3 and for use of both tobacco and alcohol, 5.6 (Table 1).

Tobacco use Prevalence was highest in the group aged 40-59 years: $42.0 \%$ (95\% Cl 40.5-43.6). In all age groups, smoking was approximately twice as common in men as in womenmale:female ratios ranged from 1.6 to 2.4 -with the smallest absolute difference observed in adolescents (4.9\%) (Table 2 ).

Smoking rates were lowest in university graduates: $25.2 \%(95 \%$ $\mathrm{Cl}$ 22.9-27.5), and both absolute and relative differences between sexes were least in these, compared to those with less education.

Smoking rates were higher in people with black skin, both women and men, than in other skin color groups, with a male:female PR of 1.4 , compared to ratios of 1.9 and 1.7 in white and mestizo persons respectively (Table 2 ).

Divorced or separated individuals more frequently reported smoking than did unmarried, married or widowed, but absolute prevalence differences by sex were least in the unmarried group. The male:female PR did not differ appreciably by marital status (range 1.8-2.3) (Table 2).

Table 1: Prevalence of tobacco and alcohol use by sex in Cuba, 2001

\begin{tabular}{|c|c|c|c|c|c|}
\hline \multirow{3}{*}{ Substance } & \multirow{2}{*}{ Total } & \multicolumn{2}{|c|}{ Gender } & \multirow{3}{*}{$\begin{array}{c}\text { M:F } \\
\text { Ratio }\end{array}$} & \multirow{3}{*}{$\begin{array}{c}\text { Absolute } \\
\text { difference } \\
\text { by sex }\end{array}$} \\
\hline & & Male & Female & & \\
\hline & $\begin{array}{r}\% \\
(95 \% \mathrm{CI})\end{array}$ & $\begin{array}{r}\% \\
(95 \% \mathrm{Cl})\end{array}$ & $\begin{array}{r}\% \\
(95 \% \mathrm{Cl})\end{array}$ & & \\
\hline Tobacco & $\begin{array}{r}30.8 \\
(30.0-31.6)\end{array}$ & $\begin{array}{r}39.9 \\
(38.1-41.2)\end{array}$ & $\begin{array}{r}22.3 \\
(20.3-24.2)\end{array}$ & 1.8 & 17.6 \\
\hline Alcohol & $\begin{array}{r}26.8 \\
(25.9-27.7)\end{array}$ & $\begin{array}{r}44.4 \\
(43.1-45.7)\end{array}$ & $\begin{array}{r}10.4 \\
(9.6-11.2)\end{array}$ & 4.3 & 34.0 \\
\hline Both & $\begin{array}{r}12.7 \\
(12.0-13.3)\end{array}$ & $\begin{array}{r}22.5 \\
(21.2-23.6)\end{array}$ & $\begin{array}{r}4.0 \\
(3.4-4.5)\end{array}$ & 5.6 & 18.5 \\
\hline
\end{tabular}

Source: ENFRENT II 
Table 2: Prevalence of tobacco use by sociodemographic variables in Cuba, 2001

\begin{tabular}{|c|c|c|c|c|c|}
\hline \multirow[b]{2}{*}{ Variable } & \multirow{2}{*}{$\begin{array}{c}\text { Total } \\
\%(95 \% \mathrm{Cl})\end{array}$} & \multicolumn{2}{|c|}{ Sex } & \multirow{2}{*}{$\begin{array}{l}\text { M:F } \\
\text { Ratio }\end{array}$} & \multirow{2}{*}{$\begin{array}{c}\text { Absolute } \\
\text { difference } \\
\text { by sex }\end{array}$} \\
\hline & & $\begin{array}{r}\text { Male } \\
\%(95 \% \mathrm{Cl})\end{array}$ & $\begin{array}{r}\text { Female } \\
\%(95 \% \mathrm{Cl})\end{array}$ & & \\
\hline \multicolumn{6}{|c|}{ Age group (years) } \\
\hline $15-19$ & $9.1(7.4-10.9)$ & $11.5(9.2-13.9)$ & $6.6(4.3-8.8)$ & 1.7 & 4.9 \\
\hline 20-39 & $28.7(27.5-29.9)$ & $37.0(35.2-38.8)$ & $20.7(19.3-22.1)$ & 1.8 & 16.3 \\
\hline $40-59$ & $42.0(40.5-43.6)$ & $52.5(50.4-54.5)$ & $32.4(30.6-34.2)$ & 1.6 & 20.1 \\
\hline$\geq 60$ & $27.4(25.7-29.0)$ & $40.4(37.8-43.0)$ & $16.5(14.7-18.3)$ & 2.4 & 23.9 \\
\hline \multicolumn{6}{|c|}{ Education completed } \\
\hline $\begin{array}{l}\text { Primary } \\
\text { school }\end{array}$ & $33.0(31.4-34.5)$ & $46.4(43.9-48.9)$ & $23.0(21.4-24.6)$ & 2.0 & 23.4 \\
\hline Middle school & $31.5(30.1-32.9)$ & $40.1(38.2-42.0)$ & $22.3(20.6-23.9)$ & 1.8 & 17.8 \\
\hline High school & $30.2(28.8-31.5)$ & $38.4(36.5-40.3)$ & $22.4(20.7-24.1)$ & 1.7 & 16.0 \\
\hline University & $25.2(22.9-27.5)$ & $30.5(27.1-34.0)$ & $20.2(17.0-23.3)$ & 1.5 & 10.3 \\
\hline \multicolumn{6}{|l|}{ Skin color } \\
\hline White & $27.9(26.9-28.8)$ & $37.2(35.9-38.4)$ & $19.3(18.2-20.4)$ & 1.9 & 17.9 \\
\hline Mestizo & $33.4(31.5-35.3)$ & $42.9(40.2-45.6)$ & $25.0(22.8-27.2)$ & 1.7 & 17.9 \\
\hline Black & $42.0(39.6-44.4)$ & $49.1(45.7-52.6)$ & $34.4(31.1-37.7)$ & 1.4 & 14.7 \\
\hline \multicolumn{6}{|l|}{ Marital status } \\
\hline Unmarried & $26.5(25.0-27.9)$ & $32.5(30.5-34.4)$ & $18.0(16.0-20.1)$ & 1.8 & 14.5 \\
\hline Married & $31.9(30.9-32.9)$ & $41.4(39.9-42.8)$ & $22.3(21.2-23.4)$ & 1.9 & 19.1 \\
\hline $\begin{array}{l}\text { Divorced or } \\
\text { separated }\end{array}$ & $38.0(35.6-40.4)$ & $55.6(51.4-59.7)$ & $30.0(27.3-32.8)$ & 1.8 & 25.6 \\
\hline Widowed & $22.8(20.0-25.6)$ & $41.4(34.6-48.3)$ & $17.9(15.0-20.9)$ & 2.3 & 23.5 \\
\hline \multicolumn{6}{|l|}{ Occupation } \\
\hline Manager & $37.6(34.4-40.9)$ & $40.8(37.1-44.5)$ & $29.8(24.0-35.6)$ & 1.4 & 11.0 \\
\hline Administrator & $29.6(25.5-33.7)$ & $38.0(31.4-44.6)$ & $25.3(20.6-29.9)$ & 1.5 & 12.7 \\
\hline $\begin{array}{l}\text { Upper-level } \\
\text { technician }\end{array}$ & $23.9(21.0-26.9)$ & $28.5(23.9-33.2)$ & $20.4(16.7-24.0)$ & 1.4 & 8.1 \\
\hline $\begin{array}{l}\text { Middle-level } \\
\text { technician }\end{array}$ & $23.9(21.0-26.9)$ & $35.5(30.6-40.4)$ & $21.3(18.0-24.5)$ & 1.7 & 14.2 \\
\hline Laborer & $42.8(41.6-44.6)$ & $45.2(43.3-47.2)$ & $30.0(26.0-34.1)$ & 1.5 & 15.2 \\
\hline $\begin{array}{l}\text { Service } \\
\text { worker }\end{array}$ & $40.2(38.0-42.5)$ & $47.0(44.1-49.9)$ & $30.0(27.0-33.0)$ & 1.6 & 17.0 \\
\hline \multicolumn{6}{|c|}{ Perceived economic situation } \\
\hline Excellent & $23.7(17.8-29.5)$ & $27.3(18.5-36.0)$ & $20.1(11.3-28.9)$ & 1.4 & 7.2 \\
\hline Good & $24.6(23.0-26.3)$ & $33.7(31.3-36.1)$ & $16.3(14.3-18.2)$ & 2.1 & 17.4 \\
\hline Fair & $30.4(29.4-31.5)$ & $39.5(38.1-41.0)$ & $21.7(20.5-22.9)$ & 1.8 & 17.8 \\
\hline Poor & $37.9(35.9-39.9)$ & $48.7(45.6-51.8)$ & $27.7(25.1-30.4)$ & 1.8 & 21.0 \\
\hline Very poor & $43.0(39.3-46.7)$ & $50.6(45.0-56.1)$ & $37.7(33.4-42.0)$ & 1.3 & 12.9 \\
\hline
\end{tabular}

Source: ENFRENT II

Overall, smoking was more prevalent in laborers, service workers and managers than in technical and administrative personnel. In male laborers, service workers and managers, smoking was more prevalent in than in upper-level technical workers. Women laborers and service workers had higher smoking prevalence than technical workers (middle- and upper-level). The lowest absolute difference in smoking prevalence by sex $(8.1 \%)$ was observed in upper-level technical workers, in whom confidence intervals for male and female prevalences overlapped, indicating a nonsignificant difference.

In both men and women, smoking prevalence varied inversely with perceived economic situation. Women smokers most frequently perceived their economic situation as very poor; the male:female ratio for that category 1.3. In contrast, in those perceiving their economic situation as excellent, confidence intervals around prevalences in men and women overlapped, indicating a non- significant difference (PR 1.4). The greatest absolute difference between male and female prevalence was in those perceiving their economic situation as poor.

Alcohol use Overall population prevalence of alcohol use in the previous 30 days was $26.8 \%$ (95\% Cl 25.9-27.7). Male:female PR was 4.3 (Table 1). The smallest male:female PR and absolute sex difference were observed in the group aged 15-19 years (PR 2.2, difference $13.4 \%$ ). Prevalence ratios ranged from 3.6 to 9.0 in the other age groups (Table 3).

Overall prevalence of alcohol use was lowest in those who had completed only primary school; this was observed in both sexes, but it was also the age group with the highest male:female PR (6.4 vs. $3.6-4.3$ in the other educational attainment groups) (Table 3).

Alcohol use was more prevalent in black than in mestizo or white Cubans. Prevalence in black women was higher than in white women, but similar to that in mestizo women (Table 3).

Patterns of alcohol use by marital status differed between the sexes. Overall prevalence was lowest in widowed Cubans $(5.8 \%, 95 \%$ Cl 4.3-7.2), and this held for both sexes (widowers 18.3\%, 95\% Cl 13.2-23.3; widows $2.5 \%, \mathrm{Cl} 1.4-3.7)$. The highest prevalence in women was in the unmarried $(14.1 \%, 95 \%$ Cl 12.3-16.0), but the confidence interval for this group overlapped with that of divorced women $(12.095 \% \mathrm{Cl}$ 9.9-14.0), which in turn overlapped with that of married women (10.0\%, 95\% Cl 9.0-10.9). In men, the married $(46.2 \%, 95 \% \mathrm{Cl} 44.7-47.8)$ and divorced (52.0\%, 95\% Cl 47.6-56.3) reported alcohol use most frequently.

There was no observable pattern of alcohol use by occupation in men and women analyzed separately, but overall prevalence was higher in managers (44.4\%, 95\% Cl 40.9-47.9) and laborers (46.6\%, 95\% Cl 44.7$48.6)$, followed by service workers $(37.0 \%, 95 \%$ CI $34.7-39.3)$. No differences were observed between administrative workers and upper- and middle-level technicians. Male:female PRs ranged from 2.9 in managers to 3.8 in laborers but there was overlap in confidence intervals.

No clear pattern emerged for perceived economic situation, neither overall nor for either sex.

Alcohol and tobacco use combined Overall, $12.7 \%$ of respondents used both tobacco and alcohol; prevalence in women was $4.0 \%$ (95\% Cl 3.4-4.5). Male:female PR was 5.6 (Table 1).

The highest overall prevalence was observed in the groups aged $40-59$ years $(16.6 \%, 95 \% \mathrm{Cl} 15.4-17.9)$, and $20-39$ years $(14.2 \%$, 
Table 3: Prevalence of alcohol use by sociodemographic variables, Cuba, 2001

\begin{tabular}{|c|c|c|c|c|c|}
\hline \multirow[b]{2}{*}{ Variable } & \multirow{2}{*}{$\begin{array}{c}\text { Total } \\
\%(95 \% \mathrm{Cl})\end{array}$} & \multicolumn{2}{|c|}{ Sex } & \multirow{2}{*}{$\begin{array}{l}\text { M:F } \\
\text { Ratio }\end{array}$} & \multirow{2}{*}{$\begin{array}{c}\text { Absolute } \\
\text { difference } \\
\text { by sex }\end{array}$} \\
\hline & & $\begin{array}{r}\text { Male } \\
\%(95 \% \mathrm{Cl}) \\
\end{array}$ & $\begin{array}{r}\text { Female } \\
\%(95 \% \mathrm{Cl}) \\
\end{array}$ & & \\
\hline \multicolumn{6}{|c|}{ Age group (years) } \\
\hline $15-19$ & $18.4(16.0-20.5)$ & $24.8(21.3-28.3)$ & $11.4(8.9-13.9)$ & 2.2 & 13.4 \\
\hline 20-39 & $33.4(32.0-34.6)$ & $53.0(51.1-54.8)$ & $14.6(13.2-15.9)$ & 3.6 & 38.4 \\
\hline $40-59$ & $28.7(27.4-30.1)$ & $49.7(47.5-51.9)$ & $9.3(8.0-10.5)$ & 5.3 & 40.4 \\
\hline$\geq 60$ & $12.6(11.2-14.0)$ & 24.3 (21.7-26.9) & $2.7(1.8-3.6)$ & 9.0 & 21.6 \\
\hline \multicolumn{6}{|c|}{ Education completed } \\
\hline $\begin{array}{l}\text { Primary } \\
\text { school }\end{array}$ & $15.9(14.7-17.1)$ & $30.9(28.6-33.1)$ & $4.8(3.8-5.7)$ & 6.4 & 26.1 \\
\hline Middle school & $29.2(27.7-20.6)$ & $46.1(44.0-48.2)$ & $10.7(9.4-12.1)$ & 4.3 & 35.4 \\
\hline High school & $31.5(30.0-33.0)$ & $50.2(47.9-52.4)$ & $13.9(12.4-15.4)$ & 3.6 & 36.3 \\
\hline University & $30.6(28.0-33.1)$ & $48.7(44.9-52.5)$ & $13.2(10.8-15.7)$ & 3.7 & 35.5 \\
\hline \multicolumn{6}{|l|}{ Skin color } \\
\hline White & $23.7(22.7-24.6)$ & $40.4(38.9-41.9)$ & $8.2(7.3-9.0)$ & 4.9 & 32.2 \\
\hline Mestizo & $30.9(29.2-32.6)$ & $49.2(46.7-51.8)$ & $14.7(12.9-16.5)$ & 3.3 & 34.5 \\
\hline Black & $36.6(34.1-39.0)$ & $56.6(53.3-60.0)$ & $14.9(12.3-17.6)$ & 3.8 & 41.7 \\
\hline \multicolumn{6}{|l|}{ Marital status } \\
\hline Unmarried & $29.5(27.9-31.1)$ & $40.5(38.3 .42 .7)$ & $14.1(12.3-16.0)$ & 2.9 & 26.4 \\
\hline $\begin{array}{l}\text { Married or } \\
\text { cohabiting }\end{array}$ & $28.2(27.1-29.2)$ & $46.2(44.7-47.8)$ & $10.0(9.0-10.9)$ & 4.6 & 36.2 \\
\hline $\begin{array}{l}\text { Divorced or } \\
\text { separated }\end{array}$ & $24.5(22.3-26.8)$ & $52.0(47.6-56.3)$ & $12.0(9.9-14.0)$ & 4.3 & 40.0 \\
\hline Widowed & $5.8(4.3-7.2)$ & 13.2-23.3) & $2.5(1.4-3.7)$ & 7.3 & 15.8 \\
\hline \multicolumn{6}{|l|}{ Occupation } \\
\hline Manager & $44.4(40.9-47.9)$ & $55.1(50.7-59.4)$ & $18.8(13.8-23.7)$ & 2.9 & 36.3 \\
\hline Administrator & $26.7(22.7-30.6)$ & $50.1(43.1-57.0)$ & $14.7(10.5-18.8)$ & 3.4 & 35.4 \\
\hline $\begin{array}{l}\text { Upper-level } \\
\text { technician }\end{array}$ & $28.5(25.5-31.5)$ & $48.3(43.5-53.0)$ & $13.1(10.1-16.1)$ & 3.7 & 35.2 \\
\hline $\begin{array}{l}\text { Middle-level } \\
\text { technician }\end{array}$ & $25.3(22.5-28.1)$ & $46.9(44.3-52.4)$ & $12.6(10.0-15.1)$ & 3.7 & 34.3 \\
\hline Laborer & $46.6(44.7-48.6)$ & $52.9(50.8-55.0$ & $14.0(10.5-17.2)$ & 3.8 & 38.9 \\
\hline $\begin{array}{l}\text { Service } \\
\text { worker }\end{array}$ & $37.0(34.7-39.3)$ & $50.1(48.0-53.8)$ & $16.0(13.2-18.8)$ & 3.1 & 34.1 \\
\hline \multicolumn{6}{|c|}{ Perceived economic situation } \\
\hline Excellent & $22.2(16.0-28.5)$ & $36.2(25.8-46.6)$ & $8.4(2.3-14.6)$ & 4.3 & 27.8 \\
\hline Good & $28.0(26.3-29.7)$ & $45.5(43.1-48.0)$ & $11.8(10.0-13.6)$ & 3.9 & 33.7 \\
\hline Fair & $27.4(26.3-28.4)$ & $45.4(43.8-46.9)$ & $10.2(9.2-11.1)$ & 4.4 & 35.2 \\
\hline Poor & $24.3(22.3-26.3)$ & $40.3(37.2-43.5)$ & $9.2(7.4-10.9)$ & 4.4 & 31.1 \\
\hline Very poor & $22.5(19.1-25.8)$ & $38.8(33.1-44.5)$ & $10.9(7.6-14.1)$ & 3.6 & 27.9 \\
\hline
\end{tabular}

Source: ENFRENT II

95\% Cl 13.3-15.1). Male:female PRs were 6.0 for the former age group and 4.6 for the latter. In women, no significant differences were observed between these age groups. Lowest prevalence was consistently seen in the oldest and youngest groups, globally and in both sexes. The youngest group had the lowest overall prevalence $(4.9 \%, 95 \% \mathrm{Cl} 3.6-6.2)$ and the lowest male:female PR (2.7) and absolute difference (4.4\%) (Table 4).

Overall prevalence of tobacco and alcohol use was higher in those who had completed middle $(14.4 \%, 95 \% \mathrm{Cl} 13.3-15.5)$ and high school $(14.0 \%, 95 \% \mathrm{Cl} 12.9-15.1)$ than in those with only primary education $(9.4 \%, 95 \% \mathrm{Cl} 8.4-10.4)$ or who had completed university $(10.9 \%, 95 \%$ Cl $9.1-12.7)$. In women, prevalence was lowest in those with only primary education $(2.1 \%, 95 \%$ Cl 1.4-2.6). Male:female PR difference was lowest in the most educated group (14.8\%).

Use of tobacco and alcohol was more common in black $(21.1 \%, 95 \% \mathrm{Cl} 18.9-23.2)$ and mestizo $(16.4 \%, 95 \% \mathrm{Cl} 14.0-16.8)$ than in white Cubans (10.3\%, 95\% Cl 9.6-10.9). The smallest absolute difference in prevalence by sex was observed in the latter ( $16.3 \%$ vs $18.3 \%$ and $25.7 \%$, in mestizo and black Cubans, respectively), although the male:female PR was the highest (7.8) where the smallest gender difference was observed. However, in black women, both addictions were reported 3 times more than in white women: $7.7 \%(5.6-9.8)$ vs. $2.4 \%(1.9-2.9)$, respectively.

By marital status, prevalence of tobacco and alcohol use was lowest among the widowed $(3.0 \%$, $95 \% \mathrm{Cl} 2.0-4.1$ ), while other marital status groups had similar prevalences of approximately $13 \%$ (unmarried $13.3 \%$, 95\% Cl 12.1-14.4; married $13.3 \%, 95 \% \mathrm{Cl} 12.5-14.0$; divorced $13.1 \%$, $95 \% \mathrm{Cl} 11.4-14.9)$. A similar pattern was seen in women, but not men, in whom prevalence was substantially higher in divorced men $(31.2 \%$, $95 \% \mathrm{Cl} 27.0-35.5)$ than in married $(22.6 \%, 95 \%$ Cl 21.3-23.9) and unmarried men (19.2\%, 95\% Cl 17.6-20.8). The lowest male:female PR was observed in the unmarried group (3.8).

By occupation, overall use of tobacco and alcohol was most common in laborers $(24.2 \%$, 95\% Cl 22.6-25.9), managers $(19.5 \%, 95 \% \mathrm{Cl}$ 16.8-22.2) and service workers $(19.4 \%, 95 \% \mathrm{Cl}$ 17.3-21.5). No important differences between occupational groups were observed in women. The lowest male:female PR was found in managers (3.2). The smallest absolute differences between men and women were observed in upper-level technical workers (13.5\%), administrative workers (15.2\%) and managers (16.7\%). In men, prevalence of tobacco and alcohol use by upper-level technicians $(17.2 \%, 95 \% \mathrm{Cl} 13.3-$ $21.1)$ was lower than that in laborers $(27.8 \%$, 95\% Cl 25.9-29.6) and service workers (27.2\%; 95\% Cl 24.3-30.1) (Table 4).

Prevalence of tobacco and alcohol use was lower overall in individuals whose perceived economic situation was excellent $(7.8 \%, 95 \% \mathrm{Cl} 3.8-11.7)$ or good $(11.0 \%, 95 \% \mathrm{Cl} 9.8-$ 12.1) than in those who reported their economic situation as fair $(12.9 \%, 95 \% \mathrm{Cl} 12.3-13.7)$ or poor $(14.0 \%, 95 \% \mathrm{Cl} 12.4-15.7)$, a pattern also observed in men (although with overlap in confidence intervals for excellent and very bad perceived economic situation). No gradient was observed in women, in whom prevalence was much lower than in men in all age groups (male:female PR 3.1-6.2).

As self-perceived economic situation improved, absolute difference in prevalence between men and women-but not male:female PR—lessened (Table 4). 
Table 4: Prevalence of combined tobacco and alcohol use by sociodemographic variables in Cuba, 2001

\begin{tabular}{|c|c|c|c|c|c|}
\hline \multirow[b]{2}{*}{ Variable } & \multirow{2}{*}{$\begin{array}{c}\text { Total } \\
\%(95 \% \mathrm{Cl})\end{array}$} & \multicolumn{2}{|c|}{ Gender } & \multirow{2}{*}{$\begin{array}{c}\text { M:F } \\
\text { Ratio }\end{array}$} & \multirow{2}{*}{$\begin{array}{l}\text { Absolute } \\
\text { difference } \\
\text { by sex }\end{array}$} \\
\hline & & $\begin{array}{r}\text { Male } \\
\%(95 \% \mathrm{Cl}) \\
\end{array}$ & $\begin{array}{r}\text { Female } \\
\%(95 \% \mathrm{CI}) \\
\end{array}$ & & \\
\hline \multicolumn{6}{|c|}{ Age group (years) } \\
\hline $15-19$ & $4.9(3.6-6.2)$ & $7.0(5.0-9.1)$ & $2.6(1.2-4.1)$ & 2.7 & 4.4 \\
\hline 20-39 & $14.2(13.3-15.1)$ & $23.8(22.2-25.3)$ & $5.2(4.2-5.9)$ & 4.6 & 18.6 \\
\hline $40-59$ & $16.6(15.4-17.9)$ & $29.3(27.2-31.3)$ & $4.9(3.9-5.9)$ & 6.0 & 24.4 \\
\hline$\geq 60$ & $6.4(5.4-7.3)$ & $13.1(11.2-15.0)$ & $0.7(0.3-1.1)$ & 18.4 & 12.4 \\
\hline \multicolumn{6}{|c|}{ Education completed } \\
\hline $\begin{array}{l}\text { Primary } \\
\text { school }\end{array}$ & $9.4(8.4-10.4)$ & $19.3(17.4-21.1)$ & $2.1(1.4-2.6)$ & 9.2 & 17.2 \\
\hline Middle school & $14.4(13.3-15.5)$ & $23.6(21.4-25.0)$ & $4.4(3.4-5.3)$ & 5.4 & 19.2 \\
\hline High school & $14.0(12.9-15.1)$ & $23.2(21.4-25.0)$ & $5.7(4.3-6.3)$ & 4.3 & 17.5 \\
\hline University & $10.9(9.1-12.7)$ & $18.5(15.3-21.6)$ & $3.7(2.3-5.1)$ & 5.0 & 14.8 \\
\hline \multicolumn{6}{|l|}{ Skin color } \\
\hline White & $10.3(9.6-10.9)$ & $18.7(17.7-19.8)$ & $2.4(1.9-2.9)$ & 7.8 & 16.3 \\
\hline Mestizo & $16.4(14.0-16.8)$ & $25.1(22.8-27.4)$ & $6.8(5.4-8.1)$ & 3.7 & 18.3 \\
\hline Black & $21.1(18.9-23.2)$ & $33.4(30.1-36.8)$ & $7.7(5.6-9.8)$ & 4.3 & 25.7 \\
\hline \multicolumn{6}{|c|}{ Marital status } \\
\hline Unmarried & $13.3(12.1-14.4)$ & $19.2(17.6-20.8)$ & $5.0(3.8-6.2)$ & 3.8 & 14.2 \\
\hline $\begin{array}{l}\text { Married or } \\
\text { cohabiting }\end{array}$ & $13.3(12.5-14.0)$ & $22.6(21.3-23.9)$ & $3.9(3.3-4.5)$ & 5.8 & 18.7 \\
\hline $\begin{array}{l}\text { Divorced or } \\
\text { separated }\end{array}$ & $13.1(11.4-14.9)$ & $31.2(27.0-35.5)$ & $4.9(3.4-6.3)$ & 6.4 & 26.3 \\
\hline Widowed & $3.0(2.0-4.1)$ & $11.2(6.9-15.6)$ & $0.9(0.3-1.5)$ & 12.4 & 10.3 \\
\hline \multicolumn{6}{|l|}{ Occupation } \\
\hline Manager & $19.5(16.8-22.2)$ & $24.4(21.0-27.9)$ & $7.7(4.3-11.0)$ & 3.2 & 16.7 \\
\hline Administrator & $10.8(8.1-13.5)$ & $20.9(15.0-26.7)$ & $5.7(2.9-8.4)$ & 3.7 & 15.2 \\
\hline $\begin{array}{l}\text { Upper-level } \\
\text { technician }\end{array}$ & $9.6(7.5-11.7)$ & $17.2(13.3-21.1)$ & $3.7(1.9-5.5)$ & 4.6 & 13.5 \\
\hline $\begin{array}{l}\text { Middle-level } \\
\text { technician }\end{array}$ & $10.7(8.7-12.7)$ & $22.6(18.0-27.1)$ & $3.7(2.2-5.2)$ & 6.1 & 18.9 \\
\hline Laborer & $24.2(22.6-25.9)$ & $27.8(25.9-29.6)$ & $5.8(3.5-8.0)$ & 4.8 & 22.0 \\
\hline $\begin{array}{l}\text { Service } \\
\text { worker }\end{array}$ & $19.4(17.3-21.5)$ & $27.2(24.3-30.1)$ & $7.6(5.5-9.6)$ & 3.6 & 19.6 \\
\hline \multicolumn{6}{|c|}{ Perceived economic situation } \\
\hline Excellent & $7.8(3.8-11.7)$ & $11.7(5.7-17.8)$ & $3.8(0.0-8.9)$ & 3.1 & 7.9 \\
\hline Good & $11.0(9.8-12.1)$ & $19.0(17.0-20.9)$ & $3.6(2.5-4.7)$ & 5.3 & 15.4 \\
\hline Fair & $12.9(12.3-13.7)$ & $22.4(21.1-23.7)$ & $3.8(3.2-4.4)$ & 5.9 & 18.6 \\
\hline Poor & $14.0(12.4-15.7)$ & $24.7(21.9-27.6)$ & $4.0(2.7-5.2)$ & 6.2 & 20.7 \\
\hline Very poor & $14.0(11.3-16.7)$ & $24.1(19.5-28.7)$ & $6.9(4.1-9.8)$ & 3.5 & 17.2 \\
\hline
\end{tabular}

Table 5: Profile of Cuban women most frequently using tobacco, alcohol or both, 2001

\begin{tabular}{|c|c|c|c|}
\hline Variable & Tobacco & Alcohol & Both \\
\hline Age (years) & $40-59$ & $15-59$ & $20-59$ \\
\hline Education completed & $<$ University & $\geq$ Middle-school & No clear pattern \\
\hline Skin color & Black & Black or mestizo & Black or mestizo \\
\hline Marital status & Divorced or separated & Not widowed & Not widowed \\
\hline Occupation & $\begin{array}{c}\text { Manager } \\
\text { Laborer } \\
\text { Service worker }\end{array}$ & No clear pattern & No clear pattern \\
\hline $\begin{array}{l}\text { Perceived } \\
\text { economic situation }\end{array}$ & Very poor & No clear pattern & No clear pattern \\
\hline $\begin{array}{l}\text { M:F prevalence } \\
\text { ratio }(P R)\end{array}$ & 1.8 & 4.3 & 5.6 \\
\hline
\end{tabular}

Source: ENFRENT II
The profile of Cuban women for tobacco and alcohol use, separately and combined, is displayed in Table 5.

\section{DISCUSSION}

A review of the Cuban literature found no nationallevel studies of men's and women's use of these two legal drugs. Although the data presented here are from 2001, a study limitation, they constitute the most complete available national data on tobacco and alcohol use in male and female urban residents.

We observed tobacco use prevalence in Cuban women to be high compared to the rest of the Americas and the world. Although prevalence varies widely internationally, in the 192 countries for which WHO reports prevalence overall and by sex, Cuba ranks among the top 33. In Latin America, Cuba's prevalence of tobacco use in women $(23.1 \%)$ is surpassed only by Chile $(36.8 \%)$ and Argentina (24.2\%). The smallest male:female differences regionally are reported in the Americas and Europe and the largest in the Eastern Pacific: data from China show a dramatic gap between the sexes: $63 \%$ in men and $4.0 \%$ in women.[26,27] There is also current debate about the possibility that women are more vulnerable to secondhand smoke, with no consensus. Some findings do not support this hypothesis[28,29] while others do find evidence of increased susceptibility in women, likely due to various factors, including genetic differences and the anti-estrogenic effect of tobacco smoke.[30,31]

Taken together, the foregoing suggests that reducing smoking among Cuban women could lower the high risk of preventable illness and death they now face from multiple diseases that share smoking as a risk factor-including cardiovascular, respiratory, neurological, endocrine, digestive and reproductive conditions as well as lung cancer and other neoplasms.[32]

The smaller difference in smoking prevalence between male and female adolescents in this study has been observed elsewhere; there is a need for more effective actions to prevent smoking in both sexes in this age group.[33]

Prevalence of alcohol use varies widely in the Americas region. In populations aged 15-64 years, for example, prevalence surpasses $50 \%$ in Argentina, Chile and Uruguay, is more than $40 \%$ in Bolivia and is close to $35 \%$ in Ecuador and Peru. Sex differences are less pronounced in the population aged 15-34 years, but prevalence in women is lower than in men.[20]

Prevalence of alcohol use in women aged 15-17 years has been reported as follows in several South American countries: Argentina 50.2\%, Chile 
$50 \%$, Colombia $63.1 \%$, Uruguay $65.3 \%$ and Brazil $66.8 \%$. Ecuador and Mexico are found in another group with a range of $39.8 \%$ to $50.0 \%$. In Bolivia, prevalence is $22.7 \%$. Lower levels of alcohol consumption were found among Cuban adolescent women, perhaps reflecting Cuba's efforts to safeguard the health and wellbeing of children and adolescents, this age group merits careful monitoring, given the regional patterns referred to above.[34]

Many lifelong behaviors are established in adolescence, so the finding that almost one in five adolescents in this study consumed alcohol should serve as an alert. The small difference between the sexes could reflect the permanent incorporation of Cuban women into spheres of social and economic activities once traditionally the realm of men: as their social role has changed, so have their behaviors. Thus, current trends are more similar to men's patterns, including in alcohol consumption, although with a certain tendency to hide or deny problems of substance abuse.[34]

We observed that tobacco use dropped as educational level increased, a finding reported in other studies whose authors argued more education enabled better understanding of smoking risks and benefits of not smoking, leading to self-protective behaviors. We saw this pattern only in men however; there was no such gradient in women, a phenomenon described by PAHO in the 1970s and confirmed in subsequent surveys, showing a tendency to higher smoking prevalence in more educated women. Our finding of similar smoking prevalence in women across all educational levels could indicate a weaker effect of educational level on smoking habits in women, suggesting a need for further research on the role of education and possible contribution of other factors.[35-37]

Higher rates of alcohol use observed in highly-educated individuals could be related to their greater purchasing power than in other educational groups.[38] We did not assess amount consumed.

The smaller difference in prevalence of tobacco use between unmarried women and men (14.5\%) could be attributed to the preponderance in this category of youth, in whom the male:female differences in tobacco use are progressively diminishing.[26]
The higher rates of alcohol use in unmarried women could be explained by research results suggesting that women may drink, in the first place, in response to loneliness, to calm the anxiety and conflicts it induces, and to alleviate pain.[39,40]

An important limitation in the analysis of the variables of "skin color" and "perceived economic situation" associated with smoking and alcohol consumption in women is limited comparability with other studies. Notably, the highest prevalence of tobacco and alcohol use, separately and together, was observed in non-white women, perhaps a reflection of other differences that could help explain these risk behaviors.

Perceived economic situation could to a certain extent reflect objective situations in other contexts, but in Cuba, the relationship is unclear and even sometimes contradictory. This lack of clarity could partly explain the lack of relationship between perceived economic situation and combined use of tobacco and alcohol by our female respondents. It is an intriguing issue and calls for further study in the Cuban population.[39,40]

Our results call attention to the particularities of women's relationship to tobacco and alcohol, which differs from men's and calls for gender-specific prevention and intervention strategies. They are also consistent with findings of studies of perception of health risks, including alcohol and tobacco, in which women reported heightened perception compared to men, providing better prospects for change toward health-promoting and -protecting behaviors.[41,42]

\section{CONCLUSIONS}

Our results provide a baseline for assessment of subsequent changes in the evolution of alcohol and tobacco use in the Cuban population, particularly in women, since it is the first study to present a profile of Cuban women's use of alcohol and tobacco. Its results may thus provide public health decision-makers with evidence to design more effective strategies to help reduce these risk behaviors and their damage to individuals, families and society. $-1 /$ -

\section{REFERENCES}

1. World Health Organization. WHO report on the global tobacco epidemic, 2008: the MPOWER package [Internet]. Geneva: World Health Organization; 2008 [cited 2011 Jan 15]. Available from: http://www.who.int/tobacco/mpower/2008/ en/index.html

2. González Menéndez R. Tabaco y alcohol: Las drogas bajo piel de cordero. Rev Cubana Med Gen Integr. 2007;23(3). Spanish.

3. Shafey O, Eriksen M, Ross H, Mackay J. El Atlas del Tabaco. 3rd ed. [place of publication unknown]: American Cancer Society; 2009. Spanish.

4. Teesson M, Baillie A, Lynskey A, Manor B, Degenhardt L. Substance use, dependence and treatment seeking in the United States and Australia: a cross-national comparison. Drug Alcohol Depend. 2006 Feb 1;81(2):149-55.

5. Mackay L, Eriksen M, Shafe O. El atlas del tabaco. 2nd ed.[place unknown]: American Cancer Society; 2006. Spanish.

6. Villalbí JR, Gual A. Las políticas preventivas del consumo de sustancias adictivas y la responsabilidad de los profesionales. Adicciones. 2009;21(1):5-8. Spanish.

7. Gual A. PortalesMedicos [Internet]. Barcelona: PortalesMedicos; c1999-2011. El alcohol es la principal causa de 60 enfermedades. Hospital Clínico Barcelona; 2006 [cited 2010 Nov 5]; [about 2 screens]. Available from: http://www. portalesmedicos.com/noticias/estudio_alcohol_ europa_061023.htm. Spanish.

8. WHO. MPOWER: un plan de medidas para hacer retroceder la epidemia de tabaquismo. Geneva: World Health Organization; 2008. 41 p. Spanish.

9. Flores LD La Mujer y el Abuso de Alcohol. Departamento de Adicciones de México. [Internet]. México. 2010 [cited 2010 Nov 5]. Available from: http://sesver.ssaver.gob.mx/pls/ portal/docs/page/inicio/adicciones/drogas/la mujer y el abuso de alcohol[1]. Spanish.

10. Campo-Arias A, Ceballos GA, Herazo E. Consumo de cigarrillos en estudiantes de una ciudad de Colombia: factores asociados al género. Rev Salud Pública (Bogotá). 2009 Aug;11(4):601-12. Spanish.

11. International Early Lung Cancer Action Program Investigators; Henschke Cl, Yip R, Miettinen OS. Women's susceptibility to tobacco carcinogens and survival after diagnosis of lung cancer. JAMA. 2006 Jul 12;296(2):180-4

12. Freedman ND, Leitzmann MF, Hollenbeck AR, Schatzkin A, Abnet CC. Cigarette smoking and subsequent risk of lung cancer in men and women: analysis of a prospective cohort study. Lancet Oncol. 2008 Jul;9(7):649-56.

13. Mackay J, Amos A. Women and tobacco. Respirology. 2003 Jun;8(2):123-30.

14. Ortega N. Consumo de alcohol, factores de riesgo e información sobre daños a la salud en estudiantes de pregrado CONADIC 2002. Observatorio mexicano en tabaco, alcohol y otras drogas. SSA México [Internet]. 2010 [cited 2010 April 5]. Available from: http:// www.portalesmedicos.com/publicaciones/ articles/2084/5/Consumo-de-alcohol\%2Cfactores-de-riesgo-e-informacion-sobreda\% F 1 os-a-la-salud-en-estudiantes-depregrado. Spanish.

15. Taylor B, Rehm J, Caldera JT, Aburto C, Bejarano J, Cayetano C, et al. Alcohol, Género, Cultura y Daños en las Américas. Reporte Final del Estudio Multicéntrico. Washington DC: Pan American Health Organization; 2007 Nov. 69 p. Spanish.

16. Samet JM, Yoon SY. Women and the Tobacco Epidemic. Challenges for the 21st Century. 1st ed. Geneva: World Health Organization; 2001.

17. National Hygiene, Epidemiology and Microbiology Institute (CU). Informe Técnico II Encuesta 
Nacional de Factores de Riesgo y Actividades Preventivas de las Enfermedades no Trasmisibles. Havana: Ministry of Public Health (CU); 2002. Spanish.

18. WHO. Estrategias e intervenciones de base fáctica para disminuir los daños relacionados con el alcohol. Evaluación mundial de los problemas de salud pública provocados por el consumo nocivo de alcohol. 60 Asamblea Mundial de la Salud, abril 2007. Geneva: World Health Organization; 2007 Apr. Spanish.

19. Varona P, Herrera D, García R, Bonet M, Romero T, Venero S. Smoking-Attributable Mortality in Cuba. MEDICC Rev. 2009 Jul;11(3):43-7.

20. Comisión Interamericana para el Control del Abuso de Drogas. Elementos orientadores para las Políticas Públicas sobre Drogas en la subregión. Primer estudio comparativo sobre consumo de drogas y factores asociados en poblaciones de 15 a 64 años de edad. New York: United Nations; 2008 Apr. 94 p. Spanish.

21. WHO. Informe sobre dependencia y abandono del tabaco. Conferencia de las Partes en el Convenio Marco de la OMS para el Control del Tabaco. FCTC/COP/3/10, agosto 2008. Geneva: World Health Organization; 2008 Aug. 17 p. Spanish.

22. Ministry of Public Health (CU). Proyecciones de la Salud Pública en Cuba para el año 2015. [Internet]. Havana: Ministry of Public Health (CU); 2006 [cited 2010 Nov 5]. Available from: http://www.salud2015.sld.cu/. Spanish.

23. Ministry of Public Health (CU). Programa Nacional de Prevención y Control del Alcoholismo y otras Farmacodependencias. Havana: Editorial Ciencias Médicas; 2005. Spanish

24. National Statistics Bureau (CU). Anuario Estadístico de Cuba 2009. Havana: National Statistics Bureau (CU); 2010. Spanish.

25. Cañizares P, Barroso U, Alfonso A, García R, Alfonso K, Chang la Rosa M, et al. Estimaciones usadas en diseños muestrales complejos: Aplicaciones en la encuesta de salud cubana en el año 2001. Rev Panam Salud Pública. 2004 Mar;15(3):176-84. Spanish.

26. WHO. Gender and tobacco control: A Policy Brief, 2007. Geneva: World Health Organization; 2007.

27. Kreuzer M, Boffetta P, Whitley E, Ahrens W, Gaborieau W, Heinrich J, et al. Gender differences in lung cancer risk by smoking: a multi- centre case-control study in Germany and Italy. Br J Cancer. 2000 Jan;82(1):227-33.

28. Thun MJ, Henley SJ, Calle EE. Tobacco use and cancer: an epidemiologic perspective for geneticists. Oncogene. 2002 Oct 21;21(48):7307-25.

29. Fu JB, Kau TY, Severson RK, Kalemkerian GP. Lung cancer in women: analysis of the national Surveillance, Epidemiology, and End Results database. Chest. 2005 Mar;127(3):768-77.

30. International Early Lung Cancer Action Program Investigators. Women's Susceptibility to Tobacco Carcinogens and Survival After Diagnosis of Lung Cancer [Internet]. JAMA. 2006 [cited 2010 Nov 5];296(2):180-4. Available from: http://jama. ama-assn.org/cgi/content/full/296/2/180

31. Centers for Disease Control and Prevention (CDC). Differences by Sex in Tobacco Use and Awareness of Tobacco Marketing - Bangladesh, Thailand, and Uruguay, 2009. MMWR. 2010 May 28:59(20):613-8.

32. WHO. International Agency for research on cancer. IARC Monographs on the Evaluation of Carcinogenic Risks to Humans. 2004;(83):1081-97.

33. Lancés L, Quesada A, Hadad J, Varona P, Landrove O. Centres for Disease Control and Prevention (CDC). Pan American Health Organization [Internet]. Washington D.C: Pan American Health Organization; c2011. Resultados de la Encuesta Mundial de Tabaquismo en la Juventud: Resultados en las Américas; 2002 [cited 2010 Nov 5]; [about 2 screens]. Available from: http://www.paho.org/ spanish/sha/be v23n2-EMTJ.htm. Spanish.

34. PAHO. Alcohol y Salud en las Américas: Un caso para la Acción. Geneva: Pan American Health Organization; 2007. Spanish.

35. Varona P. Humo ambiental de tabaco en el hogar y percepción de riesgo en la población residente en el área urbana. Cuba, año 2001 [thesis]. [Havana]: Calixto García School of Medicine; 2008. Spanish.

36. Centers for Disease Control and Prevention. Tobacco Use Among Adults-United States, 2005. MMWR [Internet]. 2006 Oct 27 [cited 2010 Nov 5];55(42):1145-8. Available from: http://www.cdc.gov/mmwr/preview/mmwrhtml/ mm5542a1.htm

37. Centers for Disease Control and Prevention. Tabaquismo y Salud en las Américas. Informe de la Cirujana General. Pan American Health Organization; 1992. Spanish
38. WHO; Department of Mental Health and Substance Abuse. Global Status Report on Alcohol 2004. Geneva: World Health Organization; 2004. 94 p.

39. Dusek D, Girdano DA. Apocatástasis.com [Internet]. [place unknown]: Apocatastasis. c2011. Causas sobre el alcoholismo en las mujeres. In: Drogas, un estudio basado en hechos. $3^{\text {rd }}$ ed; 2010 [cited 2010 Apr 16]; [about 2 screens]. Available from: http://www. apocatastasis.com/alcoholismo/femenino-mujeralcoholica.php. Spanish.

40. Boland M, Fitzpatrick P, Scallan E, Daly L, Herity B, Horgan J, et al. Trends in medical student use of tobacco, alcohol and drugs in an Irish university, 1973-2002. Drug Alcohol Depend. 2006 Nov 8;85(2):123-8.

41. Corral V, Frías A, González L. Percepción de riesgos, conducta proambiental y variables sociodemográficas en una comunidad de Sonora México. Región y Sociedad. 2003;15(26). Spanish.

42. Gustafson PE. Gender Differences in Risk Perception: Theoretical and Methodological perspectives. Risk Anal. 1998 Dec;18(6):805-11.

\section{THE AUTHORS}

Patricia Varona Pérez (Corresponding author: patricia.varona@infomed.sld.cu), physician specializing in epidemiology. Associate researcher, National Hygiene, Epidemiology and Microbiology Institute (INHEM), Havana, Cuba.

Martha Chang de la Rosa, sociologist. Adjunct researcher, INHEM, Havana, Cuba.

René G. García Roche, physician specializing in biostatistics. Adjunct researcher, INHEM, Havana, Cuba.

Mariano Bonet Gorbea, physician specializing in epidemiology. Director, INHEM, Havana, Cuba.

Submitted: June 7, 2010

Approved for publication: September 27, 2011 Disclosures: None 